\title{
Anthropometric measurements of the knee: time to make it fit
}

\author{
Emmanuel Thienpont $\cdot$ Roland Becker
}

Published online: 12 November 2014

(C) European Society of Sports Traumatology, Knee Surgery, Arthroscopy (ESSKA) 2014

Anthropometry is the study of the measurement of the human body in terms of the dimensions of bone, muscle and adipose tissue. The word derives from a composition of the Greek words anthropos or "man" and metron or "measure". It is a science which measures the range of body sizes within populations. Therefore, anthropometry plays a vital role in industrial design, clothing design, ergonomics and architecture whereby the statistical data gathered with respect to the distribution of body dimensions in a population are used to optimize product development. The most commonly studied parameters are human height, weight, organs and finally human aesthetics. For most of the parameters, it is understandable that there will be morphologic differences between different ethnic groups. Obviously, there is a difference in size between the Asian and European populations. Asians require smaller component sizes in total knee arthroplasty (TKA). The correct fit of the Duracon ${ }^{\circledR}$, Scorpio ${ }^{\circledR}$, NexGen ${ }^{\circledR}$, PFCSigma ${ }^{\circledR}$ and the UKnee ${ }^{\circledR}$ was studied in the Asian population by Jia-kuo et al. [12]. Mediolateral undersizing was found for the smaller implants, but overhang in the larger sizes.

Anthropometric dimensions for a specific population can be ranked by size and described in terms of percentiles. In furniture design, general dimensions are chosen as such so that they fit the fifth percentile of the female population and the 95th percentile for the male population. This rationale accommodates approximately $90 \%$ of the population. The

\footnotetext{
E. Thienpont $(\bowtie)$

University Hospital Saint Luc - UCL, Brussels, Belgium

e-mail: emmanuel.thienpont@uclouvain.be

R. Becker

City-Hospital Brandenburg, Brandenburg, Germany
}

question in knee surgery is of course, should an implant be designed to accommodate $90 \%$ of the population or should it cover the entire population. Early knee designs were developed "down the middle" using the mean sizes for males and females and as a result could not fit everyone perfectly.

The mismatch of femoral components, especially in the mediolateral dimension of female patients, was one of the main reasons for the need to design specific implants in order to reduce component overhang in female patients $[2,4,6]$. Gender differences were also observed in the anatomy of the trochlear groove [9]. A strong correlation between the morphology of the proximal femur and the trochlea was reported by Wright et al. [10] in their paper.

A meta-analysis on the outcome of TKA using gender implants was performed recently and showed that genderspecific TKA indeed reduces component overhang, but without any positive effect on clinical outcome [11].

However, the functional evaluation of patients after TKA revealed an overall significant number of patients (regardless of gender) who presented with mediolateral overhang of either the femoral or tibial component. Bonnin et al. [1] reported on overhang for $66 \%$ on the femoral side and $61 \%$ on the tibial side. There was also a significant negative correlation between the overall overhang and clinical outcome. An overhang of the femoral component of more than $3 \mathrm{~mm}$ appears to be associated with an almost twofold increased risk of knee pain [7].

One may presume that the mismatch between component design and bone morphology could have a significant impact on patient satisfaction. However, it remains unclear how much of a mismatch between the implant and the morphology of the knee might be tolerable as many other contributing factors might also have a significant impact on the clinical outcome as well. 
With the introduction of patient-specific instrumentation, more attention was paid to bone morphology. Patientspecific instrumentation is based on 3D MRI or CT images, and thousands of these 3D images are available for research today which gives us a unique opportunity for detailed analysis of human bone morphology. It also allows us to identify differences between ethnic groups, which could have a significant impact on component design for globally active orthopaedic companies.

Osteoarthritic changes of the knee also cause changes to the bony geometry. Significant differences were found with regards to a few parameters between the arthritic and the non-arthritic knee, as reported by Puthumanapully et al. [8] in this issue.

Tibial designs have moved more recently from the symmetric non-anatomical tibial plateau to the more anatomical plateau, showing smaller AP dimensions on the lateral side. The Natural knee ${ }^{\circledR}$, which was developed in 1985 , used already an asymmetric plateau successfully. With this design, studies showed less impingement at the area of the popliteus tendon and a better fit to the tibial plateau [5].

This finding has been confirmed recently. The coverage of the tibial bone by a non-anatomical component is about $85-87 \%$. This bony coverage increases up to $92 \%$ when anatomical designs are used [3]. The non-anatomical design requires internal rotation of more than $5^{\circ}$ for compensation in 39-60\% of patients in order to improve the bony coverage.

There seems to be a renaissance of the more asymmetric anatomical tibial design in TKA.

However, it remains questionable whether the return from the non-anatomical to the anatomical design will really improve knee function and clinical outcome.

Anthropometric data will help to improve the understanding of the bony morphology in relation to the knee.

\section{References}

1. Bonnin MP, Schmidt A, Basiglini L et al (2013) Mediolateral oversizing influences pain, function, and flexion after TKA. Knee Surg Sports Traumatol Arthrosc 21:2314-2324

2. Chin KR, Dalury DF, Zurakowski D et al (2002) Intraoperative measurements of male and female distal femurs during primary total knee arthroplasty. J Knee Surg 15:213-217

3. Dai Y, Scuderi GR, Bischoff JE, Bertin K, Tarabichi S, Rajgopal A (2014) Anatomic tibial component design can increase tibial coverage and rotational alignment accuracy: a comparison of six contemporary designs. Knee Surg Sports Traumatol Arthrosc. doi:10.1007/s00167-014-3282-0

4. Hitt K, Shurman JR, Greene K et al (2003) Anthropometric measurements of the human knee: correlation to the sizing of current knee arthroplasty systems. J Bone Joint Surg Am 85-A(Suppl 4): $115-122$

5. Kazakin A, Nandi S, Bono J (2014) Diagnosis and treatment of intraoperative popliteus tendon impingement. J Knee Surg. doi:1 $0.1055 / \mathrm{s}-0034-1367729$

6. Lonner JH, Jasko JG, Thomas BS (2008) Anthropomorphic differences between the distal femora of men and women. Clin Orthop Relat Res 466:2724-2729

7. Mahoney OM, Kinsey T (2010) Overhang of the femoral component in total knee arthroplasty: risk factors and clinical consequences. J Bone Joint Surg Am 92:1115-1121

8. Puthumanapully PK, Harris SJ, Leong A et al (2014) A morphometric study of normal and varus knees. Knee Surg Sports Traumatol Arthrosc. doi:10.1007/s00167-014-3337-2

9. Varadarajan KM, Gill TJ, Freiberg AA et al (2009) Gender differences in trochlear groove orientation and rotational kinematics of human knees. J Orthop Res 27:871-878

10. Wright SJ, Boymans TAEJ, Grimm B, Miles AW, Kessler O (2014) Strong correlation between the morphology of the proximal femur and the geometry of the distal femoral trochlea. Knee Surg Sports Traumatol Arthrosc. doi:10.1007/s00167-014-3343-4

11. Xie X, Lin L, Zhu B et al (2013) Will gender-specific total knee arthroplasty be a better choice for women?. Eur J Orthop Surg Traumatol, A systematic review and meta-analysis. doi:10.1007/ s00590-013-1396-6

12. Yang B, Song CH, Yu JK et al (2014) Intraoperative anthropometric measurements of tibial morphology: comparisons with the dimensions of current tibial implants. Knee Surg Sports Traumatol Arthrosc. doi:10.1007/s00167-014-3258-0 